\title{
Sleep after critical illness: Study of survivors of acute respiratory distress syndrome and systematic review of literature
}

\author{
Sahajal Dhooria, Inderpaul Singh Sehgal, Anshu Kumar Agrawal', Ritesh Agarwal, \\ Ashutosh Nath Aggarwal, Digambar Behera
}

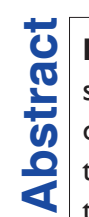

Background and Aims: This study aims to evaluate the sleep quality, architecture, sleep-related quality of life, and sleep-disordered breathing (SDB) in acute respiratory distress syndrome (ARDS) survivors early after discharge. Materials and Methods: In this prospective, observational study, consecutive patients with ARDS discharged from the Intensive Care Unit (ICU) underwent evaluation with Epworth sleepiness scale (ESS), Pittsburgh Sleep Quality Index (PSQI), Functional Outcomes of Sleep Questionnaire (FOSQ), and overnight polysomnography. Patients having one or more of the following characteristics were classified as having abnormal sleep: ESS $>10$, PSQI $>5$, FOSQ $<17.9$, apnea-hypopnea index $(\mathrm{AHI}) \geq 5$, or $\mathrm{AHI}$ during rapid eye movement (REM) sleep $\geq 5$. Results: Twenty patients (median interquartile range [IQR] age of 24 [22-28] years, I I [55\%] females) were included in the study. Acute febrile illness of unknown etiology with multi-organ dysfunction syndrome was the most common underlying etiology for ARDS. The median (IQR) $\mathrm{PaO}_{2} / \mathrm{FiO}_{2}$ ratio and APACHE II scores on admission were I 76 (I5I-19I.5) and I4 (I4-I6), respectively. The median (IQR) duration of stay in the ICU was 10 days (7.3-19.5). The overall sleep efficiency (median [IQR], 54\% [32.3-65.4\%]) was poor. None of the patients had ESS >10, seven (35\%) had global PSQI $>5$ and one had FOSQ < 17.9. Ten (50\%) patients had at least one characteristic that suggested abnormal sleep (4 insomnia, 2 central sleep apnea, I obstructive sleep apnea, I REM-SDB, and 2 with a high PSQI, but no specific sleep abnormality). Conclusions: Sleep disturbances are common in ARDS survivors early after discharge from the ICU.

Keywords: Acute respiratory distress syndrome, critically ill, Intensive Care Unit, mechanical ventilation, obstructive sleep apnea, sleep apnea

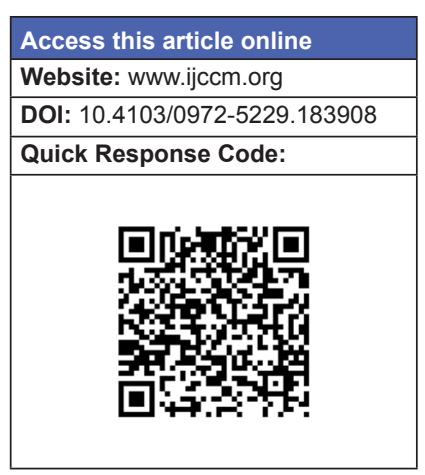

\section{Introduction}

Sleep disturbances are encountered in critically ill patients admitted to the Intensive Care Unit (ICU). ${ }^{[1]}$ The etiology is multifactorial including the acute illness itself, noisy environment of the ICU, exposure to light during the night, interruptions due to patient care-related

\section{From:}

Department of Pulmonary Medicine, Postgraduate Institute of Medical Education and Research, Chandigarh, ${ }^{1}$ Department of Gastroenterology, Govind Ballabh Pant Institute of Postgraduate Medical Education and Research, New Delhi, India

Correspondence:

Dr. Sahajal Dhooria, Department of Pulmonary Medicine, Postgraduate Institute of Medical Education and Research, Chandigarh - 160 012, India.

E-mail: sahajal@gmail.com activities, altered circadian rhythm, medications, and others. ${ }^{[1-6]}$ Patients with the acute respiratory distress syndrome (ARDS) have severe acute illness, require mechanical ventilation in a majority of the instances.

This is an open access article distributed under the terms of the Creative Commons Attribution-NonCommercial-ShareAlike 3.0 License, which allows others to remix, tweak, and build upon the work non-commercially, as long as the author is credited and the new creations are licensed under the identical terms.

For reprints contact: reprints@ @medknow.com

How to cite this article: Dhooria S, Sehgal IS, Agrawal AK, Agarwal R, Aggarwal AN, Behera D. Sleep after critical illness: Study of survivors of acute respiratory distress syndrome and systematic review of literature. Indian J Crit Care Med 2016;20:323-31. 
They are predisposed to the risk of patient-ventilator dyssynchrony and frequently require sedatives and other psychotropic medications. All these factors make them a unique population, especially prone to sleep disturbances. Severe disruptions have been reported in the sleep architecture in this subgroup of patients. ${ }^{[7]}$ Sleep disturbance that arises during the stay in the ICU may continue long after discharge..$^{[8]}$ Survivors of ARDS suffer from many adverse physical and psychological sequelae and decreased quality of life. ${ }^{[9]}$ Chronic sleep disorders such as insomnia and sleep apnea present several months after discharge in these patients and contribute to the impaired quality of life. ${ }^{[8,10]}$

Sleep architecture has been previously studied in ARDS patients during the stay in the ICU. ${ }^{[7]}$ Chronic sleep disorders have been described in the survivors of ARDS several months after discharge. ${ }^{[8,10]}$ Sleep disturbances have also been studied in the general population of critically ill patients both in the early and late postdischarge periods. ${ }^{[11]}$ We wanted to determine the prevalence of sleep disturbances diagnosed on the basis of a composite criterion in a uniform study population (of subjects with ARDS) early in the postdischarge period. In this study, we describe the changes in sleep quality, sleep-related quality of life, sleep architecture, and the prevalence of sleep-disordered breathing (SDB) in the survivors of ARDS early after discharge. We also perform a systematic review of studies describing sleep disturbances in patients discharged from ICUs after recovery from critical illness (with or without ARDS).

\section{Materials and Methods}

This was a prospective observational study conducted between July 2011 and September 2012 in the Department of Pulmonary Medicine of this institute. All patients provided informed consent and the study protocol was approved by the Ethics Review Committee.

\section{Study participants}

Patients were included in the study if they satisfied all the following criteria: (i) Age 18-65 years, (ii) ARDS defined by the American-European Consensus Conference criteria, ${ }^{[12]}$ (iii) ICU stay of at least $48 \mathrm{~h}$ duration, (iv) intubated and mechanically ventilated for $\geq 24 \mathrm{~h}$, (v) successfully extubated and discharged from the respiratory ICU after weaning from mechanical ventilation and survived for $\geq 1$ month, and (vi) provided an informed consent to participate in the study. Patients with any of the following characteristics were excluded: (i) Known psychiatric, neurological, cognitive, or sleep disorder prior to the present illness, (ii) receiving any sedative or psychotropic medication after discharge from the ICU, (iii) discharge after getting tracheostomized, and (iv) failure to provide an informed consent. Patients were enrolled and all the study procedures were conducted at 1 month of discharge from the ICU.

\section{Study setting and procedures}

The respiratory ICU comprises eight beds, has a nurse: Patient ratio of 1:2, and is overseen by a team of six pulmonary and critical care consultants. The nursing care is provided by a qualified nurse with assistance from the hospital attendants under the direct supervision of the doctors on duty (two pulmonary fellows at all times throughout the day and night). All patients are subjected to appropriate laboratory investigations and therapeutic management during their stay according to the ICU protocols. ${ }^{[13,14]}$

Demographic details (age, gender, and body mass index [BMI]) were recorded for all the included patients. The etiology of ARDS and the $\mathrm{PaO}_{2} / \mathrm{FiO}_{2}$ ratio at presentation were noted. All of them underwent endotracheal intubation and mechanical ventilation. Assist-control (volume controlled) mode of ventilation was used for all patients during the initial phase according to the ARDSNet protocol whereas pressure support ventilation was employed during the weaning period. ${ }^{[15]}$ The duration of ICU stay and mechanical ventilation, the dose and duration of sedatives and neuromuscular blockers, and the respective cumulative doses were recorded. The severity of the illness was assessed with the APACHE II score calculated within $24 \mathrm{~h}$ of ICU stay.

Measures of sleepiness, sleep quality, and sleep-related quality of life

All patients completed the Epworth Sleepiness Scale (ESS), a self-administered questionnaire which rates the likelihood of falling asleep (from 0 to 3 ) under eight different daily life situations. ${ }^{[16]}$ The maximum score is 24 and a score above 10 indicates significant daytime sleepiness. The patients also responded to the Pittsburgh Sleep Quality Index (PSQI), a scale used for the subjective assessment of sleep quality. ${ }^{[17]}$ The questionnaire consists of 19 items with responses arranged on an integer scale from 0 to 3, which assesses seven components of sleep quality: Subjective sleep quality, latency, duration, and efficiency; sleep disturbances, sleeping medication use, and daytime dysfunction. The total sleep quality score, obtained by adding the component scores, ranges between 0 and 21, with higher scores representing lower sleep quality. A score above 5 indicates poor sleep quality. The scale can be adapted to enable the subject to respond verbally to items on the scale by having an interviewer read the statement to the subjects. Finally, 
the study participants also completed the Functional Outcomes of Sleep Questionnaire (FOSQ), which comprises 30 items, each item coded on a five-point scale (from 0 to 4) ${ }^{[18]}$ It assesses the impact of excessive sleepiness in five domains: Activity level, vigilance, intimacy and sexual relationships, general productivity, and social outcome. The total score ranges from 5 to 20 and a score of more than 17.9 is used as the threshold for defining a normal sleep-related quality of life. ${ }^{[19]}$ All the questionnaires were in English. All study participants completed the questionnaires under supervision (and if needed with some help from the interviewer), following a detailed explanation.

\section{Polysomnography}

An overnight polysomnography (PSG) was performed on all study subjects within a month of discharge from the ICU. Monitoring of the electroencephalogram using two frontal, two central, and two occipital channels (placed according to the international 10-20 system), electro-oculogram, submental and anterior tibialis electromyogram, electrocardiogram (lead I), chest and abdominal movements, airflow (using oronasal thermal sensor and nasal air pressure transducer), and pulse oximetry was performed. Sleep scoring was done according to 2007 American Academy of Sleep Medicine criteria. ${ }^{[20]}$ Apnea-hypopnea index (AHI) was calculated for each patient. The total sleep duration, duration of each sleep stage, and sleep efficiency were calculated. AHI during rapid eye movement (REM) sleep (AHI-REM) was also separately calculated. SDB was defined by $\mathrm{AHI} \geq 5$, while SDB-REM was defined by an AHI-REM $\geq 5$.

Patients having one or more of the following characteristics were classified as having abnormal sleep: ESS $>10$, PSQI $>5$, FOSQ $<17.9$, AHI $\geq 5$, or AHI-REM $\geq 5$.

\section{Statistical analysis}

Statistical analysis was performed using the commercial statistical package SPSS (Version 22 for MS-Windows , IBM Corp., Armonk, New York, USA). Continuous data are expressed as mean (standard deviation [SD]) or median (interquartile range [IQR]), based on the Shapiro-Wilk test of normality, whereas categorical data are expressed as number with percentage. Differences in continuous variables between two groups were compared using the Mann-Whitney U-test. $P<0.05$ was considered statistically significant.

\section{Results}

A total of 360 patients were admitted to the respiratory ICU during the study period; 74 (20.6\%) had a diagnosis of ARDS, of which 50 (67.6\%) patients survived their ICU stay. Of these, eight fell outside the age limits of the study, five had known psychiatric and/or sleep disorders, three were managed on noninvasive ventilation, five patients were discharged on a tracheostomy, four patients died within 1 month of discharge, and five patients refused to provide informed consent. Finally, twenty patients (median [IQR] age 24 [22-28] years, 11 [55\%] females) were enrolled in this study [Table 1]. Acute febrile illness of unknown etiology with multi-organ dysfunction syndrome was the most common underlying etiology for ARDS. The median (IQR) $\mathrm{PaO}_{2} / \mathrm{FiO}_{2}$ ratio and APACHE II scores were 176 (151-191.5) and 14 (14-16), respectively. The median (IQR) duration of stay in the ICU was 10 (7.3-19.5) days. Midazolam (14 [70\%] patients) and vecuronium (12 [60\%] patients) were the most commonly employed sedative and neuromuscular blocking agents, respectively.

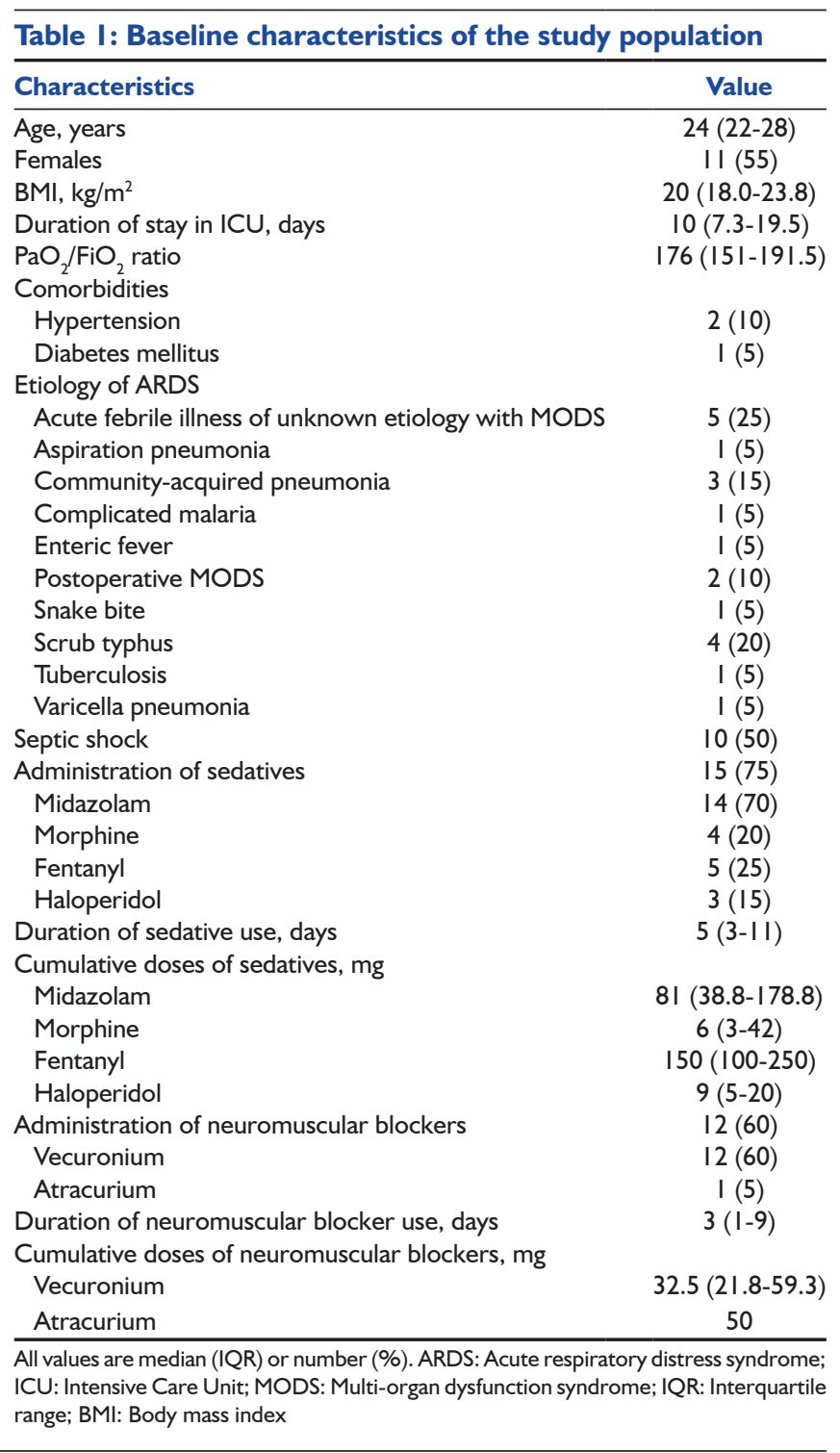


None of the patients had an ESS score (median [IQR] score of 6 [3.3-7]) of more than 10 [Table 2]. Seven $(35 \%)$ patients had a global PSQI above the threshold score of 5 , representing poor overall sleep quality. The mean (SD) global FOSQ was 18.37 (0.62), with only one patient having a FOSQ score below the conventionally used cutoff of 17.9. Ten (50\%) patients did not respond to the sexual and intimacy component of the FOSQ scale. The overall sleep efficiency (median [IQR], 54\% [32.3-65.4\%]) was poor [Table 3]. Most of the sleep duration comprised stage 1 and 2, while slow wave and REM sleep together accounted for $21.4 \%$ (median) of the total sleep duration. The median (IQR) AHI was $1.9(0.7-2.7)$. Three (15\%) and six (30\%) patients had $\mathrm{AHI}$ and AHI-REM $\geq 5$, respectively [Table 3]. Overall, ten $(50 \%)$ of the twenty patients had at least one of the following characteristics signifying abnormal sleep: ESS $>10$, PSQI $>5$, FOSQ $<17.9$, AHI $\geq 5$, or AHI-REM $\geq 5$. Of these, four had insomnia (all had complaints of difficulty initiating sleep), two had central sleep apnea, and one had obstructive sleep apnea (OSA). In one patient, REM SDB was the predominant abnormality detected. In two other patients, the PSQI was high; however, no specific sleep abnormality could be identified. On comparing the characteristics of subjects with $(n=3)$ and without $(n=17) \mathrm{SDB}$ (i.e. AHI $\geq 5)$, those with SDB had a longer duration of receiving sedation in the ICU $(P=0.048)$ with higher cumulative doses of midazolam $(P=0.044)$ administered.

\section{Discussion}

This study demonstrates that at least one parameter representing abnormal sleep is present in half of the mechanically ventilated ARDS patients early after discharge from the ICU. In this study, we adopted a multi-pronged approach in an attempt to capture the different aspects of sleep disturbances including daytime sleepiness (using ESS), sleep quality (using PSQI), sleep-related quality of life (using FOSQ), and specific sleep abnormalities such as SDB (using PSG). To our knowledge, this is the first study in which an unselected group of ARDS patients has been evaluated early in the post-ICU discharge period, irrespective of the presence or absence of any sleep-related complaints.

We searched the PubMed and EMBASE databases for relevant studies describing the occurrence of sleep disturbances after recovery from critical illness (with or without ARDS) using the following search terms: ("sleep disordered breathing" OR "sleep apnoea" OR "sleep apnoea" OR "sleep quality" OR "sleep-related quality of life" OR "sleep disorders" OR "sleep disturbances") AND ("acute respiratory distress syndrome" OR "ards"
Table 2: Daytime sleepiness, sleep quality, and sleep-related quality of life as assessed by Epworth Sleepiness Scale, Pittsburgh Sleep Quality Index, and Functional Outcomes of Sleep Questionnaire in study subjects

\begin{tabular}{lc}
\hline Item & Score \\
\hline ESS & $6(3.3-7)$ \\
ESS > I0 & 0 \\
PSQI & $\mathrm{I}(0.25-\mathrm{I})$ \\
Component I (subjective sleep quality) & $\mathrm{I}(\mathrm{I}-2)$ \\
Component 2 (sleep latency) & $\mathrm{I}(0-\mathrm{I})$ \\
Component 3 (sleep duration) & $0(0)$ \\
Component 4 (habitual sleep efficiency) & $\mathrm{I}(\mathrm{I}-\mathrm{I})$ \\
Component 5 (sleep disturbances) & $0(0-0)$ \\
Component 6 (use of sleep medication) & $\mathrm{I}(\mathrm{I}-2)$ \\
Component 7 (daytime dysfunction) & $5(4-7)$ \\
Global PSQI & $7(35)$ \\
Patients with PSQI $>5, n$ (\%) & \\
FOSQ, mean \pm SD & $3.75(3.62-3.75)$ \\
General productivity & $3.80(3.63-3.80)$ \\
Vigilance & $3.75(3.50-4.00)$ \\
Social outcome & $3.56(3.44-3.67)$ \\
Activity level & $3.75(3.69-3.75)$ \\
Intimacy & $18.50(I 8.09-18.73)$ \\
Global FOSQ, mean \pm SD & $\mathrm{I}(5)$ \\
Global FOSQ < I7.9, $n$ (\%)
\end{tabular}

All values are median (IQR) unless stated otherwise. SD: Standard deviation; IQR: Interquartile range; ESS: Epworth Sleepiness Scale; PSQI: Pittsburgh Sleep Quality Index; FOSQ: Functional Outcomes of Sleep Questionnaire

Table 3: Polysomnographic characteristics and type of sleep abnormality in the study participants

\begin{tabular}{lc}
\hline Characteristics & Value \\
\hline Total sleep time, min & $278.5(20 I .8-383$. I) \\
Sleep latency, min & $21.5(8.4-6 I .0)$ \\
Sleep efficiency, percentage of total time in bed & $54(32.3-65.4)$ \\
Sleep stages, percentage of total sleep time & \\
Stage I & $24.4(16.6-48.5)$ \\
Stage 2 & $45.8(23.3-60.9)$ \\
Slow wave sleep & $15.9(8.4-24 . I)$ \\
REM & $5.5(2.3-15.1)$ \\
AHI & $1.9(0.7-2.7)$ \\
AHI $\geq 5$ & $3(15)$ \\
AHI-REM $\geq 5$ & $6(30)$ \\
Oxygen saturation while awake & $98(97-99)$ \\
Minimum oxygen saturation during sleep & $94(85-95)$ \\
Desaturation index & $2.3($ I-6.8) \\
Sleep abnormality & \\
Insomnia & $4(20)$ \\
Central sleep apnea & $2(10)$ \\
Obstructive sleep apnea & I (5) \\
REM sleep-disordered breathing & I (5) \\
High PSQI without specific sleep abnormality & $2(10)$ \\
\hline All values are median (IQR) or number (\%). AHI: Apnea-hypopnea index; PSQI: Pittsburgh \\
Sleep Quality Index; REM: Rapid eye movement; IQR: Interquartile range; PSQI: Pittsburgh \\
Sleep Quality Index; ESS: Epworth Sleepiness Scale
\end{tabular}

OR "adult respiratory distress syndrome" OR "acute lung injury" OR "critical illness" OR "critically ill" OR "intensive care" OR "ICU" OR "mechanical ventilation" OR "mechanically ventilated"). From the EMBASE database, citations under only the two categories, "articles" and "articles in press" were included. We reviewed the list of references of original studies, editorials, and 
reviews; and also sifted through our personal files. We excluded case reports, abstracts, comments, editorials, and reviews; studies describing the quality of sleep or sleep disturbances during ICU stay; studies describing the outcomes of patients with known sleep disorders (e.g., OSA); studies performed only in the pediatric population; and studies performed in noncritically ill hospitalized patients or in postoperative subjects.

The initial database search yielded 991 studies, of which 17 studies (2314 subjects) met the inclusion criteria [Figure 1 and Table 4]. ${ }^{[8,11,21-35]}$ The instruments used to assess sleep disorders/disturbances varied widely from unstructured questionnaires to specific instruments, while PSG was performed in only four studies. ${ }^{[8,23,29,31]}$ Most studies described the proportion of patients having poor sleep quality, sleep disturbances, or sleep-related symptoms, while the studies in which PSG was performed also reported sleep efficiency, sleep latency, sleep architecture, and/or AHI [Table 5]. Fourteen studies reported the percentage of patients with abnormal sleep (subjective or objective). ${ }^{[8,11,21,23,24,26-28,30-35]}$ Six of these were performed early $(<1$ month) and five late $(>1$ month) after discharge while three studies reported on both early and late outcomes [Table 6]. The median (range) percentage of patients having abnormal sleep was higher in the early period (66.7\% [40.4-80\%]) than in the late period (38.5\% [6.4-57\%]).

\begin{tabular}{|c|c|c|c|c|c|c|}
\hline Author (year) & Country & Design & Type of ICU & $\begin{array}{l}\text { Number } \\
\text { of patients }\end{array}$ & Patient population & $\begin{array}{l}\text { Instruments used } \\
\text { to assess sleep }\end{array}$ \\
\hline Hall-Smith et al. $(1997)^{[21]}$ & United Kingdom & Prospective & Not mentioned & 26 & ICU stay $>5$ days & $\begin{array}{l}\text { Unstructured, client-led } \\
\text { interview }\end{array}$ \\
\hline Niskanen (1999) ${ }^{[22]}$ & Finland & Prospective & Medical-surgical & 368 & $\begin{array}{l}\text { At least one ICU admission lasting } \\
\geq 96 \mathrm{~h}\end{array}$ & Finnish version of the NHP \\
\hline Chishti et al. $(2000)^{[23]}$ & United Kingdom & Prospective & Medical-surgical & 15 & $\begin{array}{l}\text { Received }>48 \mathrm{~h} \text { of mechanical } \\
\text { ventilation in ICU }\end{array}$ & $\begin{array}{l}\text { Overnight, multi-channel } \\
\text { pneumographic studies }\end{array}$ \\
\hline Dimopoulou et al. $(200 \mathrm{I})^{[24]}$ & Greece & Prospective & Cardiac surgical & 16 & $\begin{array}{l}\text { Cardiac surgery patients with an } \\
\text { unexpected cardiac arrest in the } \\
\text { immediate postoperative period }\end{array}$ & $\begin{array}{l}\text { A specific questionnaire } \\
\text { based on the NHP }\end{array}$ \\
\hline Combes et al. $(2003)^{[25]}$ & France & Prospective & Medical-surgical & 87 & $\begin{array}{l}\text { Consecutive patients mechanically } \\
\text { ventilated for } \geq 14 \text { days in ICU }\end{array}$ & French version of the NHP \\
\hline Strahan et al. $(2003)^{[26]}$ & Northern Ireland & Prospective & Medical-surgical & 170 & $\begin{array}{l}\text { Patients with an ICU stay }>24 \mathrm{~h} \\
\text { and discharged to the general ward }\end{array}$ & $\begin{array}{l}\text { Original questionnaire } \\
\text { developed by investigators }\end{array}$ \\
\hline Granja et al. $(2005)^{[27]}$ & Portugal & Prospective & Medical-surgical & 373 & $\begin{array}{l}\text { All adult ICU patients } 6 \text { months } \\
\text { after discharge }\end{array}$ & Original questionnaire \\
\hline Strahan et al. $(2005)^{[28]}$ & United Kingdom & Prospective & Surgical & 10 & $\begin{array}{l}\text { Patients shifted to the wards } \\
\text { from ICU }\end{array}$ & Phenomenological approach \\
\hline BaHammam (2006) $)^{[29]}$ & Saudi Arabia & Prospective & Cardiac & 20 & $\begin{array}{l}\text { Consecutive patients with } \\
\text { a first-ever acute ST segment } \\
\text { elevation myocardial infarction } \\
\text { admitted to the coronary care unit }\end{array}$ & PSG \\
\hline Orwelius et al. (2008) $)^{[30]}$ & Sweden & Prospective & Medical-surgical & 497 & $\begin{array}{l}\text { Consecutive admitted adults with } \\
\text { ICU stay }>24 \mathrm{~h} \text {, and alive at } 6 \\
\text { months of hospital discharge }\end{array}$ & $\begin{array}{l}\text { Swedish version of the Basic } \\
\text { Nordic Sleep Questionnaire }\end{array}$ \\
\hline Lee et al. $(2009)^{[8]}$ & Canada & Prospective & Medical-surgical & 109 & $\begin{array}{l}\text { Survivors of acute respiratory } \\
\text { distress syndrome after discharge } \\
\text { from the ICU }\end{array}$ & $\begin{array}{l}\text { Sleep history questionnaire, } \\
\text { two Insomnia Severity } \\
\text { Index questionnaires, ESS, } \\
\text { overnight PSG }\end{array}$ \\
\hline Fanfulla et al. $(201 \mathrm{I})^{[3]]}$ & Italy & Prospective & Medical-surgical & 22 & $\begin{array}{l}\text { Patients shifted from the ICU to } \\
\text { a step down unit }\end{array}$ & 24 h PSG \\
\hline McKinley et al. $(20 I 2)^{[32]}$ & Australia & Prospective & Medical-surgical & 186 & $\begin{array}{l}\text { Mechanically ventilated for } \\
>24 \mathrm{~h} \text { with ICU stay } \geq 48 \mathrm{~h} \text {, and } \\
\text { discharged home }\end{array}$ & $\begin{array}{l}\text { I5D HRQOL instrument } \\
\text { with a five-point scale }\end{array}$ \\
\hline McKinley et al. $(2013)^{[! 1]}$ & Australia & Prospective & Medical-surgical & 199 & $\begin{array}{l}\text { ICU stay of } \geq 2 \text { nights with no } \\
\text { known history or evidence of sleep } \\
\text { disorder }\end{array}$ & $\begin{array}{l}\text { Richards Campbell Sleep } \\
\text { Questionnaire, Pittsburgh } \\
\text { Sleep Quality Index }\end{array}$ \\
\hline Vesz et al. $(20 \mid 3)^{[33]}$ & Brazil & Prospective & Medical-surgical & 79 & $\begin{array}{l}\text { Admitted to and discharged from } \\
\text { the ICU with stay } \geq 72 \mathrm{~h}\end{array}$ & ESS \\
\hline Choi et al. $(20 \mid 4)^{[34]}$ & United States & Prospective & Medical & 33 & $\begin{array}{l}\text { Admitted to a medical ICU, } \\
\text { on mechanical ventilation for } \\
\geq 4 \text { consecutive days }\end{array}$ & $\begin{array}{l}\text { Modified Given Symptom } \\
\text { Assessment Tool }\end{array}$ \\
\hline Soh et al. $(2014)^{[35]}$ & Malaysia & Prospective & Not mentioned & 104 & $\begin{array}{l}\text { Ventilated, with stay }>24 \mathrm{~h} \text {, with } \\
\text { no gross neurological deficit and no } \\
\text { history of psychotic episodes }\end{array}$ & $\begin{array}{l}\text { Questionnaire adapted } \\
\text { from Granja et al. } .^{[27]}\end{array}$ \\
\hline
\end{tabular}


Table 5: Time point of assessment and sleep disturbances reported in the included studies

\begin{tabular}{|c|c|c|}
\hline Author (year) & Time point of assessment & Sleep disturbances \\
\hline Hall-Smith et al. (1997) $)^{[21]}$ & 3 months after hospital discharge & $3(\mathrm{II} .5 \%)$ patients had poor sleep \\
\hline Niskanen et al. (1999) ${ }^{[22]}$ & 6 months after discharge & $\begin{array}{l}\text { Sleeping disturbances more frequent among patients undergoing open-heart } \\
\text { surgery or having respiratory failure than those with neurosurgical } \\
\text { disease }(P<0.0 \text { I and } P<0.00 \text { I, respectively). } \\
\text { Less common among younger patients }(18-34 \text { years) than those older } \\
\text { (55-64 years and } 65-74 \text { years; } P<0.0 \text { I for both) }\end{array}$ \\
\hline Chishti et al. $(2000)^{[23]}$ & $\begin{array}{l}\text { Within } 72 \mathrm{~h} \text { of discharge from the } \\
\text { ICU }\end{array}$ & $\begin{array}{l}\text { II }(73.3 \%) \text { patients had abnormal apnea/hypopnea index (range } 5-34 \text { events } / \mathrm{h} \text { ). } \\
\text { Time spent in some form of abnormal breathing pattern varied between } 6 \text { and } \\
41 \% \text { of the study period }\end{array}$ \\
\hline Dimopoulou et al. $(200 \mathrm{I})^{[24]}$ & 4 years postdischarge & $6(38 \%)$ patients had mild sleep disturbances, such as early awaking \\
\hline Combes et al. et al. $(2003)^{[25]}$ & $\begin{array}{l}\text { Cross-sectional study (various time } \\
\text { points) }\end{array}$ & $\begin{array}{l}\text { Mean NHP scores for sleep significantly higher than general population and } \\
\text { higher in ARDS patients as compared to the rest }\end{array}$ \\
\hline Strahan et al. $(2003)^{[26]}$ & $87 \%$ of questionnaires completed in & I I 4 (67\%) patients experienced "trouble getting to sleep" \\
\hline
\end{tabular}

Granja et al. (2005) ${ }^{[27]}$

Strahan and Brown (2005) ${ }^{[28]}$

BaHammam (2006) ${ }^{[2]}$

Orwelius et al. (2008) $)^{[30]}$

Lee et al. (2009) $)^{[8]}$

Fanfulla et al. $(201 \mathrm{I})^{[31]}$

10.2 \pm 8.7 days after admission

McKinley et al. $(2012)^{[32]}$

McKinley et al. $(2013)^{[1]}$

Vesz et al. $(2013)^{[33]}$

Choi $(2014)^{[34]}$

Soh et al. (2014) $)^{[35]}$
Weeks I, 8, and 26 after hospital discharge (sleep data available for week I and 26)

I-2 days after transfer to ward and 2 and 6 months after discharge During $I^{\text {st }}$ week following discharge from ICU

$\leq 2$ weeks and at 2 and 4 months after ICU discharge

Within 3 days of discharge from ICU
Sleep disturbances in 153 (4I\%) patients

$8(80 \%)$ patients described symptoms including tiredness, sleep difficulties, and sleep disorders

Mean sleep efficiency: $62 \%$ at 3 days and around $81.4 \%$ at 6 months

Mean sleep latency: $24.9 \mathrm{~min}$ at 3 days, $19.6 \mathrm{~min}$ at 6 months

$38 \%$ had difficulties falling asleep, $20 \%$ had poor sleep quality, and $39 \%$ reported sleep deficit

Seven (6.4\%) survivors reported persistent sleep-related symptoms

Difficulty initiating and maintaining sleep: 5, parasomnia: I, frequent nightmares: I ESS median (range): 8.5 (2-16)

Sleep efficiency (median [range]): $80.2 \%$ (50.8-95.0\%)

Sleep latency (median [range]): I 4.9 (2.1-174.I) min

Slow wave sleep (median [range]): $23.5 \%$ (7.0-32.3\%)

REM sleep (median [range]): $19.7 \%$ (5.6-24.3\%)

$10(45.5 \%)$ patients showed reduced sleep efficiency ( $<70 \%), 6$ patients had a reduced REM percentage or no REM sleep, and 3 had reduced or absent slow wave sleep

In spontaneously breathing patients: Slow wave sleep (mean): $31.9 \%$, REM sleep (mean): $14.2 \%$

Moderate (disturbed sleep or feeling I have not slept enough) to severe (sleep is almost impossible even with full use of sleeping pills or staying awake most of the night) sleep problems in 50\% at week I and $31 \%$ at week 26

Poor sleep quality in $68 \%$ after I-2 nights on the ward and in $62 \%$ and $57 \%$ at 2 and 6 months, respectively, after discharge

$43.3 \%$ with ESS score $\geq 10$

Disturbed sleep at $\geq 2$ weeks: $66.7 \%, 2$ months: $64.3 \%, 4$ months: $46.2 \%$

Sleep disturbances in 42 (40.4\%) patients

ARDS: Acute respiratory distress syndrome; ICU: Intensive Care Unit; MI: Myocardial infarction; NHP: Nottingham health profile; REM: Rapid eye movement; ESS: Epworth Sleepiness Scale

Databases searched: PubMed and EmBase

Search Terms: ("sleep disordered breathing" OR "sleep apnea" OR "sleep apnoea" OR "sleep quality" OR "sleep related quality of life" OR "sleep disorders" OR "sleep disturbances") AND ("acute respiratory distress syndrome" OR "ards" OR "adult respiratory distress syndrome" OR "acute lung injury" OR "critical illness" OR "critically ill" OR "intensive care" OR "ICU" OR "mechanical ventilation" OR "mechanically ventilated")

Citations found after initial search: 991

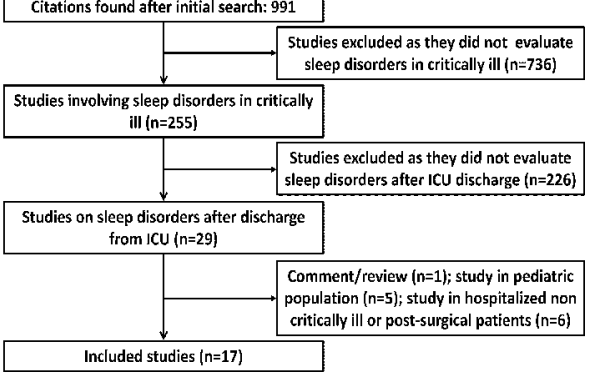

Figure I: Citation selection process for the systematic review
For the present study, we selected a specific subgroup of subjects among the patient population in the ICU, i.e., those with ARDS. This group is relatively uniform since nearly all these patients require invasive mechanical ventilation and many of them have multi-organ dysfunction. A previous study on sleep disturbances in ARDS survivors showed that $6.4 \%$ of the subjects reported persistent sleep-related symptoms 6 months or more following discharge from the ICU. ${ }^{[8]}$ In contrast, in the present study where consecutive ARDS survivors were included much earlier in the postdischarge period (within a month), a remarkably higher prevalence (50\%) of abnormal sleep was found. One contributing factor 


\begin{tabular}{|c|c|}
\hline Author (year) & $\begin{array}{l}\text { Percentage of patients } \\
\text { with abnormal sleep (\%) }\end{array}$ \\
\hline \multicolumn{2}{|l|}{ Early } \\
\hline Chishti et al. (2000) $)^{[23]}$ & 73.3 \\
\hline Strahan et al. $(2003)^{[26]}$ & 67 \\
\hline Strahan and Brown $(2005)^{[28]}$ & 80 \\
\hline Fanfulla et al. $(201 \mathrm{I})^{[31]}$ & 45.5 \\
\hline McKinley et al. $(20 / 2)^{[32]}$ & 50 \\
\hline McKinley et al. $(2013)^{[1]]}$ & 68 \\
\hline Vesz et al. $(2013)^{[33]}$ & 43.3 \\
\hline Choi et al. $(2014)^{[34]}$ & 66.7 \\
\hline Soh et al. $(2014)^{[35]}$ & 40.4 \\
\hline Median (range) & $66.7(40.4-80)$ \\
\hline \multicolumn{2}{|l|}{ Late } \\
\hline Hall-Smith et al. $(1997)^{[21]}$ & 11.5 \\
\hline Dimopoulou et al. $(200 \mathrm{I})^{[24]}$ & 38 \\
\hline Granja et al. $(2005)^{[27]}$ & 41 \\
\hline Orwelius et al. $(2008)^{[30]}$ & 39 \\
\hline Lee et al. $(2009)^{[8]}$ & 6.4 \\
\hline McKinley et al. $(20 \mid 2)^{[32]}$ & 31 \\
\hline McKinley et al. $(2013)^{[11]}$ & 57 \\
\hline Choi et al. $(2014)^{[34]}$ & 46.2 \\
\hline Median (range) & $38.5(6.4-57)$ \\
\hline
\end{tabular}

for finding a high prevalence of abnormal sleep is that a broad definition of abnormal sleep was considered in the present study. We have used multiple parameters (3 questionnaires and PSG) so as to be able to capture a higher proportion of patients with any type of sleep disturbance/reduced sleep quality/reduced sleep-related quality of life. The prevalence of abnormal sleep found in this study is consistent with the results of the systematic review in which $40-80 \%$ of critically ill patients had evidence of sleep disturbances within a month of discharge from the ICU. The most common sleep disorder was insomnia as in other studies. ${ }^{[8,26,30]}$

The reason why sleep disruption is so common early in the recovery period is uncertain. While it is well known that many environmental factors such as sound, light, ventilator, and patient care activities disrupt sleep in patients during their stay in the ICU, it is possible that the sleep pattern associated with critical illness and admission to the ICU may continue to persist for some period after discharge. ${ }^{[1,11]}$ This type of sleep disruption originating during ICU admission possibly extends into the recovery period and gradually resolves in a proportion of the patients as time elapses. As evident from the systematic review, the proportion of patients reported to have sleep disruption was higher in the early period (median 67\%), climbing down to $39 \%$ in the late period. The findings from the present study and the systematic review suggest that greater attention must be paid to sleep parameters during the early follow-up period of ARDS patients discharged from the ICU.
Sleep disturbances should be specifically looked for and managed accordingly; this might help in improving the sleep-related quality of life.

Seven of our patients had a global PSQI higher than the threshold value of 5 suggesting a poor overall sleep quality. Yet none of our patients had an ESS $>10$ suggesting that ESS that assessed only subjective excessive daytime sleepiness may be a poor measure of assessing sleep disturbances in this population. The PSQI questionnaire is more comprehensive and assesses the sleep quality based on seven different components, and hence is more robust. ${ }^{[17]} \mathrm{A}$ previous study that employed the PSQI also found that poor sleep quality in a high proportion $(70 \%)$ of patients at 1-2 day after discharge from the ICU. ${ }^{[11]}$ Contrarily, the global FOSQ was higher than 17.9 in most of the patients in our study, suggesting a good sleep-related quality of life. One of the possible reasons for this incongruity is that half of our patients did not respond to the sexual and intimacy component of the scale as sex-related issues are considered taboo for a large number of individuals in our community.

SDB was observed in only $3(15 \%)$ of the patients in the current study, in contrast to the findings of Chishti et al., in which $73 \%$ of the patients had AHI $>5 .{ }^{[23]}$ The potential reason for the discrepancy is that in studies of ICU survivors, it is not possible to ascertain whether the SDB was preexisting or appeared after the acute illness. Furthermore, the prevalence of SDB is higher with increasing age and BMI. ${ }^{[36]}$ In the present study, both the median age (24 years) and the median BMI $\left(20.0 \mathrm{~kg} / \mathrm{m}^{2}\right)$ of the patients were low. Due to this reason, the subjects in our study had a lower probability of having preexisting OSA, although factors different from BMI and age (such as craniofacial characteristics) might still play a role. In our study, we found that patients with SDB had a significantly longer duration of receiving sedation in the ICU with higher cumulative doses of midazolam. Whether a preexisting SDB led to prolonged requirement of mechanical ventilation or SDB appeared as a result of prolonged sedation and higher doses of midazolam cannot be ascertained. Besides, the number of subjects with SDB in this study is too few for a definite conclusion.

We found low sleep efficiency (median 54\%) in survivors of ARDS similar to the findings in previous studies. ${ }^{[8,29,31]}$ Further, most sleep occurred in stage 1 and 2 in our patients with markedly reduced restorative sleep (REM and slow wave sleep) as in previous reports. ${ }^{[8,31]}$ This again probably reflects the persistence of sleep abnormalities such as reduced REM and slow wave sleep that originate during the ICU stay. ${ }^{[37]}$ The finding 
of a high prevalence (30\%) of REM-related SDB in ARDS survivors needs further confirmation in a larger study cohort.

Our study has a few limitations including a small sample size. The study population mainly consists of predominantly young individuals with tropical illnesses as the cause of ARDS, thus the results may not be generalizable to other patient groups. We did not follow-up the patients over time; therefore, we cannot draw conclusions on whether the early changes in sleep quality lead to any symptomatic sleep disruption over a longer period of time. The strengths of our study include the prospective data collection in an unselected group of ARDS patients soon after discharge from the ICU.

\section{Conclusions}

Our study found a high prevalence of abnormal sleep in survivors of ARDS early after discharge from the ICU. The prevalence of sleep abnormalities found is lesser in studies performed late as compared to the early postdischarge period as is evident from the systematic review.

\section{Acknowledgment}

The authors fondly remember and heartily thank Late Prof. Dheeraj Gupta, Department of Pulmonary Medicine, Chandigarh, India, for overseeing this study.

\section{Financial support and sponsorship Nil.}

\section{Conflicts of interest}

There are no conflicts of interest.

\section{References}

1. Freedman NS, Kotzer N, Schwab RJ. Patient perception of sleep quality and etiology of sleep disruption in the intensive care unit. Am J Respir Crit Care Med 1999;159(4 Pt 1):1155-62.

2. Hilton BA. Quantity and quality of patients' sleep and sleep-disturbing factors in a respiratory intensive care unit. J Adv Nurs 1976;1:453-68.

3. Broughton R, Baron R. Sleep patterns in the intensive care unit and on the ward after acute myocardial infarction. Electroencephalogr Clin Neurophysiol 1978;45:348-60.

4. Meyer T.J, Eveloff SE, Bauer MS, Schwartz WA, Hill NS, Millman RP. Adverse environmental conditions in the respiratory and medical ICU settings. Chest 1994;105:1211-6.

5. Freedman NS, Gazendam J, Levan L, Pack AI, Schwab RJ. Abnormal sleep/wake cycles and the effect of environmental noise on sleep disruption in the intensive care unit. Am J Respir Crit Care Med 2001;163:451-7

6. Bosma K, Ferreyra G, Ambrogio C, Pasero D, Mirabella L, Braghiroli A, et al. Patient-ventilator interaction and sleep in mechanically ventilated patients: Pressure support versus proportional assist ventilation. Crit Care Med 2007;35:1048-54.

7. Cooper AB, Thornley KS, Young GB, Slutsky AS, Stewart TE,
Hanly PJ. Sleep in critically ill patients requiring mechanical ventilation. Chest 2000;117:809-18.

8. Lee CM, Herridge MS, Gabor JY, Tansey CM, Matte A, Hanly PJ. Chronic sleep disorders in survivors of the acute respiratory distress syndrome. Intensive Care Med 2009;35:314-20.

9. Herridge MS, Tansey CM, Matté A, Tomlinson G, Diaz-Granados N, Cooper A, et al. Functional disability 5 years after acute respiratory distress syndrome. N Engl J Med 2011;364:1293-304.

10. Parsons EC, Kross EK, Caldwell ES, Kapur VK, McCurry SM, Vitiello MV, et al. Post-discharge insomnia symptoms are associated with quality of life impairment among survivors of acute lung injury. Sleep Med 2012;13:1106-9.

11. McKinley S, Fien M, Elliott R, Elliott D. Sleep and psychological health during early recovery from critical illness: An observational study. J Psychosom Res 2013;75:539-45.

12. Bernard GR, Artigas A, Brigham KL, Carlet J, Falke K, Hudson L, et al. The American-European consensus conference on ARDS. Definitions, mechanisms, relevant outcomes, and clinical trial coordination. Am J Respir Crit Care Med 1994;149(3 Pt 1):818-24.

13. Agarwal R, Aggarwal AN, Gupta D, Behera D, Jindal SK. Etiology and outcomes of pulmonary and extrapulmonary acute lung injury/ARDS in a respiratory ICU in North India. Chest 2006;130:724-9.

14. Agarwal R, Srinivasan A, Aggarwal AN, Gupta D. Adaptive support ventilation for complete ventilatory support in acute respiratory distress syndrome: A pilot, randomized controlled trial. Respirology 2013;18:1108-15.

15. Ventilation with lower tidal volumes as compared with traditional tidal volumes for acute lung injury and the acute respiratory distress syndrome. The Acute Respiratory Distress Syndrome Network. N Engl J Med 2000;342:1301-8.

16. Johns MW. Daytime sleepiness, snoring, and obstructive sleep apnea. The Epworth sleepiness scale. Chest 1993;103:30-6.

17. Buysse DJ, Reynolds CF $3^{\text {rd }}$, Monk TH, Berman SR, Kupfer DJ. The pittsburgh sleep quality index: A new instrument for psychiatric practice and research. Psychiatry Res 1989;28:193-213.

18. Weaver TE, Laizner AM, Evans LK, Maislin G, Chugh DK, Lyon K, et al. An instrument to measure functional status outcomes for disorders of excessive sleepiness. Sleep 1997;20:835-43.

19. Weaver TE, Maislin G, Dinges DF, Bloxham T, George CF, Greenberg $\mathrm{H}$, et al. Relationship between hours of CPAP use and achieving normal levels of sleepiness and daily functioning. Sleep 2007;30:711-9.

20. Iber C, Ancoli-Israel S, Chesson A Jr., Quan SF. The AASM Manual for the Scoring of Sleep and Associated Events: Rules, Terminology and Technical Specifications. Westchester, IL: American Academy of Sleep Medicine; 2007.

21. Hall-Smith J, Ball C, Coakley J. Follow-up services and the development of a clinical nurse specialist in intensive care. Intensive Crit Care Nurs 1997;13:243-8.

22. Niskanen M, Ruokonen E, Takala J, Rissanen P, Kari A. Quality of life after prolonged intensive care. Crit Care Med 1999;27:1132-9.

23. Chishti A, Batchelor AM, Bullock RE, Fulton B, Gascoigne AD, Baudouin SV. Sleep-related breathing disorders following discharge from intensive care. Intensive Care Med 2000;26:426-33.

24. Dimopoulou I, Anthi A, Michalis A, Tzelepis GE. Functional status and quality of life in long-term survivors of cardiac arrest after cardiac surgery. Crit Care Med 2001;29:1408-11.

25. Combes A, Costa MA, Trouillet JL, Baudot J, Mokhtari M, Gibert C, et al. Morbidity, mortality, and quality-of-life outcomes of patients requiring $>_{\mathrm{or}}=14$ days of mechanical ventilation. Crit Care Med $2003 ; 31: 1373-81$.

26. Strahan E, Mccormick J, Uprichard E, Nixon S, Lavery G. Immediate follow-up after ICU discharge: Establishment of a service and initial experiences. Nurs Crit Care 2003;8:49-55.

27. Granja C, Lopes A, Moreira S, Dias C, Costa-Pereira A, Carneiro A, et al. Patients' recollections of experiences in the intensive care unit may affect their quality of life. Crit Care 2005;9:R96-109.

28. Strahan EH, Brown RJ. A qualitative study of the experiences of patients following transfer from intensive care. Intensive Crit Care Nurs 2005;21:160-71. 
29. BaHammam A. Sleep quality of patients with acute myocardial infarction outside the CCU environment: A preliminary study. Med Sci Monit 2006;12:CR168-72.

30. Orwelius L, Nordlund A, Nordlund P, Edéll-Gustafsson U, Sjöberg F. Prevalence of sleep disturbances and long-term reduced health-related quality of life after critical care: A prospective multicenter cohort study. Crit Care 2008;12:R97.

31. Fanfulla F, Ceriana P, D'Artavilla Lupo N, Trentin R, Frigerio F, Nava S. Sleep disturbances in patients admitted to a step-down unit after ICU discharge: The role of mechanical ventilation. Sleep $2011 ; 34: 355-62$

32. McKinley S, Aitken LM, Alison JA, King M, Leslie G, Burmeister E, et al. Sleep and other factors associated with mental health and psychological distress after intensive care for critical illness. Intensive Care Med 2012;38:627-33.

33. Vesz PS, Costanzi M, Stolnik D, Dietrich C, de Freitas KL, Silva LA, et al. Functional and psychological features immediately after discharge from an intensive care unit: Prospective cohort study. Rev Bras Ter Intensiva 2013;25:218-24.

34. Choi J, Hoffman LA, Schulz R, Tate JA, Donahoe MP, Ren D, et al. Self-reported physical symptoms in intensive care unit (ICU) survivors: Pilot exploration over four months post-ICU discharge. J Pain Symptom Manage 2014;47:257-70.

35. Soh KL, Soh KG, Ibrahim NA, Ahmad NZ, Japar S, Raman RA. Recalling ICU experiences: Patients' perspectives. Middle East J Sci Res 2014;19:106-11.

36. Ralls FM, Grigg-Damberger M. Roles of gender, age, race/ethnicity, and residential socioeconomics in obstructive sleep apnea syndromes. Curr Opin Pulm Med 2012;18:568-73.

37. Pisani MA, Friese RS, Gehlbach BK, Schwab RJ, Weinhouse GL, Jones SF. Sleep in the intensive care unit. Am J Respir Crit Care Med 2015;191:731-8. 